\title{
Toward Building a Database of Bifunctional Probes for the MS3D Investigation of Nucleic Acids Structures
}

\author{
Qingrong Zhang, Eizadora T. Yu, and Katherine A. Kellersberger \\ University of Maryland Baltimore County, Baltimore, Maryland, USA \\ Elizabeth Crosland \\ Villa Julie College, Stevenson, Maryland, USA
}

\author{
Daniele Fabris \\ University of Maryland Baltimore County and University of Maryland, Marlene and Stewart, Greenbaum \\ Cancer Center, Baltimore, Maryland, USA
}

\begin{abstract}
This report illustrates the approaches employed to investigate critical aspects of the activity of crosslinking reagents toward nucleic acid substrates, which should be evaluated to identify candidate probes for mass spectrometric 3D (MS3D) investigations of biomolecules and macromolecular complexes. Representative members of different classes of bifunctional reagents were taken into consideration, including bikethoxal and phenyl-diglyoxal [bis-(1,2dicarbonyls)], cisplatin (coordinative binding agents), chlorambucil and nitrogen mustard [bis-(2-chloroethyl)amines], and sym-triazine trichloride (triazines). Nanospray-Fourier transform mass spectrometry (FTMS) was applied without desalting or separation procedures to characterize the covalent products obtained by probing dinucleotide and trinucleotide substrates under a variety of experimental conditions in vitro. The carefully controlled composition of these substrates enabled us to obtain valid comparisons of probe activity toward individual nucleotides and evaluate possible base-specific effects, including the stability of the different adducts in solution under the selected reaction conditions. The gas-phase behavior of the observed products was investigated using sustained off-resonance irradiation collision-induced dissociation (SORI-CID) to obtain valuable information for guiding the design of sequencing experiments and helping the data interpretation. Structured RNA substrates, such as HIV-1 stemloop 1, were finally employed to investigate the structural determinant of adduct formation and highlight the different nature of the spatial information provided by the various candidate probes. (J Am Soc Mass Spectrom 2006, 17, 1570-1581) (C) 2006 American Society for Mass Spectrometry
\end{abstract}

$\mathrm{M}$ ass spectrometric 3D (MS3D) approaches seek to obtain information about the three-dimensional structure of biomolecules using chemical crosslinking and footprinting reagents followed by MS analysis [1-4]. The very wide range of applicability offered by these techniques is rapidly advancing MS3D to the forefront of the new technologies developed for the structural elucidation of biomolecules that exceed the size accessible by NMR, or afford inadequate crystallization. Fulfilling this potential, however, will require expanding the repertoire of available probes and developing new computational tools for the interpretation and utilization

Published online July 27, 2006

Address reprint requests to Dr. D. Fabris, Department of Chemistry and Biochemistry, University of Maryland, 1000 Hilltop Circle, Baltimore County, Baltimore, MD 21250, USA. E-mail: fabris@umbc.edu of this type of information in biomolecular modeling. For this purpose, a bioinformatics infrastructure has been recently created to support the efforts of investigators engaged in the development of enabling technologies for MS3D (http://ms3d.org) [5].

The MS3D investigation of nucleic acids and proteinnucleic acid complexes can count on mono- and bifunctional alkylating reagents to reveal the location of base pairs, tertiary interactions, and inter-molecular contacts $[4,6,7]$. The ability of such reagents to support the elucidation of complex RNA structures was recently tested by completing the determination of two ribosome-frameshifting pseudoknots of retroviral origin [8]. Validation of the MS3D approach was obtained by comparing the high-resolution coordinates available for one of the substrates in the Protein Data Bank with the corresponding all-atoms model generated entirely 

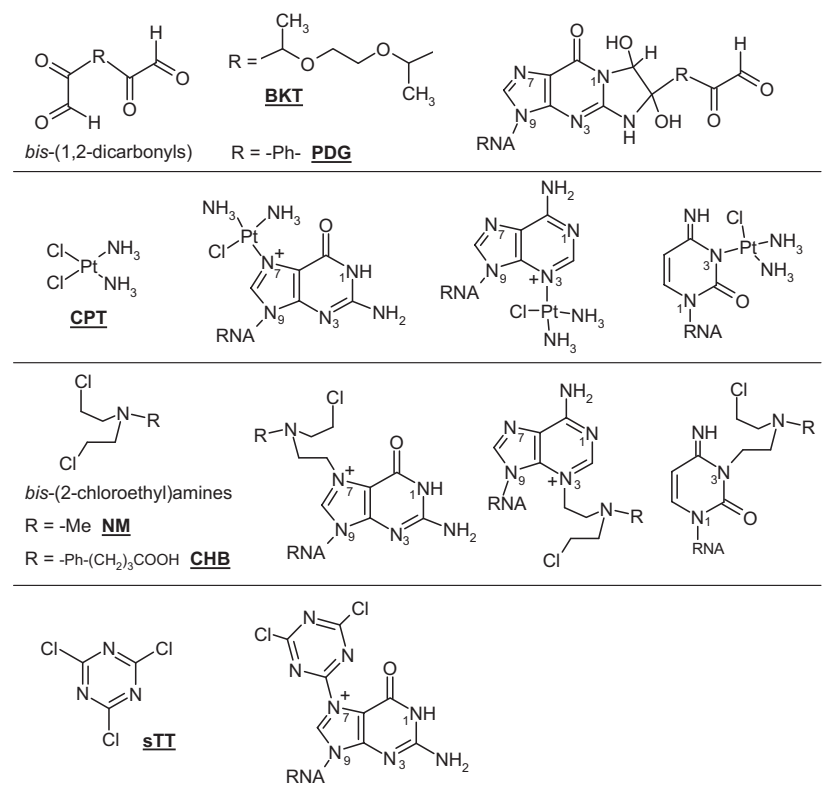

Scheme 1. Candidate bifunctional crosslinkers included in the study and putative monofunctional adduct structures: bikethoxal (BKT); phenyl-diglyoxal (PDG); cisplatin (CPT); nitrogen mustard (NM); chlorambucil (CHB); and sym-triazine trichloride (sTT).

from probe constraints. Significantly, important information necessary to model these structures was provided by the bifunctional reagent nitrogen mustard (NM) $[9,10]$ (Scheme 1) for its ability to determine the reciprocal positions of distal nucleotides that were placed in close proximity by the pseudoknot tertiary fold. The great value of this type of distance constraint in the modeling of large nucleic acid substrates, in which long-range interactions and inter-domain contacts determine the overall global fold, constitutes a powerful motivation for the investigation of alternative reagents with different base specificities and bridging distances as possible bifunctional probes for MS3D applications.

Over the years, mass spectrometry has been employed directly or coupled with high-performance separation techniques to characterize a variety of covalent adducts produced by alkylation of nucleic acids in vivo and in vitro [11-19]. The combination of direct infusion electrospray ionization (ESI) [20] and Fourier transform mass spectrometry (FTMS) [21] constitutes an ideal platform for the analysis of this type of samples, which affords the benefits of high mass accuracy and resolving power [22]. Furthermore, this platform enables the implementation of infrared multiphoton dissociation (IRMPD) [23, 24] and electron capture dissociation (ECD) $[25,26]$ for the gas-phase activation of progressively larger precursor ions, which is expected to greatly facilitate the application of top-down strategies to the characterization of probed biomolecules [27, 28]. In our hands, ESI-FTMS has proven to be particularly well suited for the direct analysis of stable DNA and
RNA adducts formed by classic monofunctional footprinting reagents $[4,6,7]$.

In an effort directed toward building a database of crosslinking agents for MS3D investigations, we have employed nanoelectrospray [29] and FTMS to evaluate the possible application of various bifunctional reagents to selected nucleic acid substrates. Candidate probes were chosen not only from the collection of established DNA/RNA structural probes that have been traditionally associated with polyacrylamide gel electrophoresis (PAGE) [30-32], but also from other classes of alkylating molecules that are widely used in cancer therapy for their ability to introduce intra- or interstrand crosslinks $[33,34]$. Small and generally unstructured substrates were employed to establish baseline reactivity profiles in the absence of steric concerns and facilitate the detection of possible base-specific effects. Factors affecting the activity of the different probes and the stability of their respective adducts were evaluated under a variety of experimental conditions, with the ultimate goal of optimizing the parameters for structural probing and product characterization. In addition, structured substrates were employed to determine the ability of candidate probes to provide different spatial constraints for the modeling of complex nucleic acid structures. This report illustrates the methods employed for evaluating the different aspects of crosslinking activity and describes the results provided by the more promising bifunctional reagents for the MS3D investigation of nucleic acids.

\section{Experimental}

\section{Reagents and Chemicals}

cis-Diamminedichloroplatinum (II) (cisplatin, CPT), bis(2chloroethyl)methylamine (nitrogen mustard, mechlorethamine, NM), 4-[bis-(2-chloroethyl)amino]benzenebutanoic acid (chlorambucil, $\mathrm{CHB}$ ), and sym-triazine trichloride (cyanuric acid, sTT) were purchased from Sigma-Aldrich Chemical Co. (St. Louis, MO) and used without further purification. Ethylene glycol bis-[3-(2-ketobutyraldehyde)ether] (bikethoxal, BKT) and 1,4-diacetalbenzene (phenyl-diglyoxal, PDG) were synthesized in house according to established procedures [35]. Due to their known mutagenic activity, these reagents were treated using all possible precautions to avoid inhalation and direct contact with eyes, mucoses, and skin, including the use of latex gloves, face-mask, and fume-hood.

Deoxyribo- and ribonucleotide dimers and trimers of homogeneous base composition were obtained from the W. M. Keck Foundation Biotechnology Resource Facility (New Haven, CT) and used with no further purification. A dimerization-deficient mutant of HIV-1 stemloop 1 (SL1A, obtained by replacing $G$ with $A$ in position 20) was obtained by in vitro transcription of the corresponding synthetic DNA template according to the phage T7 polymerase reaction [36]. Transcribed RNAs were purified by denaturing gel electrophoresis 
performed on $20 \%$ (wt/vol) polyacrylamide gels. The products of interest were recovered by electroelution from the manually excised bands. Extensive desalting was performed by ultrafiltration using Centricon devices from Millipore (Bedford, MA).

\section{Probing Reactions}

Stock solutions of individual di- and trinucleotides were prepared by dissolving the lyophilized materials obtained from the manufacturer in the selected buffer solution. The final concentrations were verified by UV absorbance at $260 \mathrm{~nm}$ using accepted values of molar absorptivity. Volatile buffers including ammonium acetate, bicarbonate, borate, and cacodylate with concentrations ranging between 10 and $100 \mathrm{mM}$ were tested in the study. Substrate mixtures of di- and trinucleotides of desired composition were prepared by mixing appropriate volumes of the initial stocks to obtain a typical $0.25 \mathrm{mM}$ concentration of each species in a final $20 \mu \mathrm{L}$ volume.

Crosslinker solutions were prepared immediately before use by weighing out the dry material and dissolving it in the most appropriate solvent compatible with their solubility. For instance, dimethylformamide was used for CPT and PDG, dioxane for sTT, acetonitrile for CHB, dimethylsulfoxide for NM, and ethanol for BKT. Appropriate volumes were then added to the substrate mixture to obtain the desired probe/substrate ratio. This was calculated by taking into account the number of potentially reactive functional groups in the crosslinker (generally two, except for sTT that has three possible alkylating sites) and the number of susceptible sites in each substrate component. A range of 1:10 to $100: 1$ probe/substrate ratios was explored in the study. Reactions were incubated at $37^{\circ} \mathrm{C}$ in a water bath for up to $24 \mathrm{~h}$ and aliquots were secured at predetermined intervals to identify the time necessary to reach completion.

\section{Probing and Mapping of SL1A}

The SL1A RNA construct was refolded before use by incubating in $10 \mathrm{mM}$ ammonium cacodylate $(\mathrm{pH} 6.0$ for CPT and $\mathrm{pH} 8.0$ for NM crosslinking) for five minutes at $90{ }^{\circ} \mathrm{C}$, followed by quick-cooling on ice to room temperature. An approximate 1:3 probe/substrate ratio (calculating all susceptible bases regardless of their accessibility in the actual construct) was used for the crosslinking reactions, with typical solutions containing a final concentration of $50 \mu \mathrm{M}$ SL1A and $250 \mu \mathrm{M}$ crosslinker in a $30 \mu \mathrm{L}$ volume. Reactions were carried out at $37^{\circ} \mathrm{C}$ for $24 \mathrm{~h}$ for CPT and $2 \mathrm{~h}$ for NM. Reactions were terminated by ethanol precipitation, which served also to dispose of the excess unreacted reagent.

Aliquots containing $0.6-2.0 \mathrm{nmol}$ of native and probed RNA were submitted to digestion with ribonuclease A (RNase A) or T1 (RNase T1). Typical digestion reactions involved 0.02-0.05 unit of enzyme per $\mathrm{mg}$ of
RNA substrate in $20 \mathrm{mM}$ ammonium acetate ( $\mathrm{pH}$ 7.5) for $30 \mathrm{~min}$ at $37^{\circ} \mathrm{C}$. Samples were stored at $-20^{\circ} \mathrm{C}$ until immediately before analysis by nano-electrospray ionization mass spectrometry.

\section{Mass Spectrometry}

Appropriate aliquots of the different reaction mixtures were diluted with water/methanol/formic acid (49: 49:2) for detection in positive ion mode, or $10 \mathrm{mM}$ ammonium acetate/isopropanol (9:1) for negative ion mode. Dilution factors were adjusted to provide final concentrations of $10 \mu \mathrm{M}$ total substrate. All analysis were performed by nanospray on a Bruker Daltonics (Billerica, MA) Apex III 7.0T Fourier transform mass spectrometer equipped with a heated-metal desolvation capillary, which was kept at $120-150{ }^{\circ} \mathrm{C}$. Each experiment required loading $\sim 5 \mu \mathrm{L}$ analyte solution into a nanospray needle with a stainless steel wire inserted from the back end to provide the necessary voltage.

For tandem experiments, precursor ions of interest were isolated in the FTMS cell using correlated rf sweeps (CHEF) [37], followed by activation through sustained off-resonance irradiation (SORI) [38]. Frequency offsets below and above the resonant frequency of the precursor ions were sampled to avoid incurring in possible "blind spots" in the product spectra [39]. Best results were achieved by using irradiation frequencies that were $600-2000 \mathrm{~Hz}$ below that of the precursor ion. Argon was used as the collision gas. All data were acquired in broadband mode and were processed using XMASS 7.0.8 (Bruker).

\section{Results and Discussion}

This investigation was prompted by the need to expand and diversify the collection of crosslinking agents for MS3D applications to include probes with alternative base specificity and bridging distance. Representative members of different classes of nucleic acid-active reagents were evaluated as possible MS3D probes (Scheme 1), including bis-(1,2-dicarbonyls) (bikethoxal, BKT, and phenyl-diglyoxal, PDG) [35, 40, 41], coordinative binding agents (cisplatin, CPT) [42, 43], bis-(2chloroethyl)amines (chlorambucil, CHB, and nitrogen mustard, NM) [44-46], and triazine derivatives (symtriazine trichloride, sTT) [47]. These candidates were selected according to their potential for reacting with relatively high yield and specificity, forming welldefined and recognizable products that should be stable under the conditions necessary for effective structural probing.

\section{Testing the Activity Characteristics of Probe Candidates}

Covalent modifications of nucleic acids are frequently studied in vitro using substrates of heterogeneous base 
composition and length (e.g., calf thymus DNA), which are treated with the chemical of interest under carefully controlled conditions. The reaction mixture is subsequently subjected to nuclease or chemical hydrolysis to reduce the size of alkylated products, often to the mononucleotide level, which facilitates their complete characterization. Alternative strategies employ ad hoc combinations of mono-, di-, or trinucleotides of predetermined base composition to allow for direct adduct analysis with no need for strand hydrolysis or sequencing procedures. In the case of candidate structural probes, accurately known substrate mixtures constitute very convenient tools for testing bifunctional crosslinkers under a variety of experimental conditions. In addition to enabling the direct characterization of reaction products, this approach can provide unbiased information about their activity toward competing substrates, which is expected to highlight base-specific effects.

This approach can be exemplified by the results of a typical reaction completed by treating equimolar amounts of homo-dinucleotides [i.e., $\mathrm{d}(\mathrm{ApA}), \mathrm{d}(\mathrm{CpC})$, $\mathrm{d}(\mathrm{GpG}), \mathrm{d}(\mathrm{TpT})$, and $\mathrm{UpU}$ ] with the nitrogen mustard agent CHB in $10 \mathrm{mM}$ ammonium acetate (pH 8.0) (see the Experimental section). The nanospray-FTMS spectra obtained in negative ion mode from a control dinucleotide mixture and from a reaction sample incubated for $24 \mathrm{~h}$ at $37^{\circ} \mathrm{C}$ are shown in Figure 1a and b, respectively. Characteristic covalent adducts were immediately identified for $\mathrm{d}(\mathrm{GpG}), \mathrm{d}(\mathrm{ApA})$, and $\mathrm{d}(\mathrm{CpC})$ (Species $\mathrm{a}, \mathrm{b}$, and $c$ in Figure $1 b$ ) corresponding to the monofunctional addition of $\mathrm{CHB}$ with formal elimination of $\mathrm{HCl}$ and hydrolysis of the remaining 2-chloroethyl function. In contrast, no adducts of $\mathrm{d}(\mathrm{TpT})$ and $\mathrm{UpU}$ were observed in the product mixture, thus confirming that the target specificity of CHB under the selected conditions was consistent with typical reactivity profiles of nitrogen mustard agents (Scheme 1) [45, 46].

A rigorous quantitative approach to determine the yields of probing reactions would require the availability of appropriate standards, or should take in account the expected variations of ionization efficiency between reactants and products. The ESI behavior of nucleic acid analytes is sensitive to the hydrophobicity and gasphase proton affinity dictated by their base composition [48], as demonstrated here by the fact that individual homo-dinucleotides offered widely different signal intensities despite their equimolar concentrations in the standard solution (Figure 1a). The effects of chemical modifications on these characteristics may not be readily predictable, but they are likely to be substantial for adducts of small and singly-charged substrates, such as the di- and trinucleotides employed in these experiments. For this reason, a safe estimate of probe reactivity was obtained by determining the percentage of reactant consumption, based on signal intensities detected for the susceptible dinucleotide at the beginning and at the end of the probing reaction. Using inert dinucleotides in the product mixture as convenient
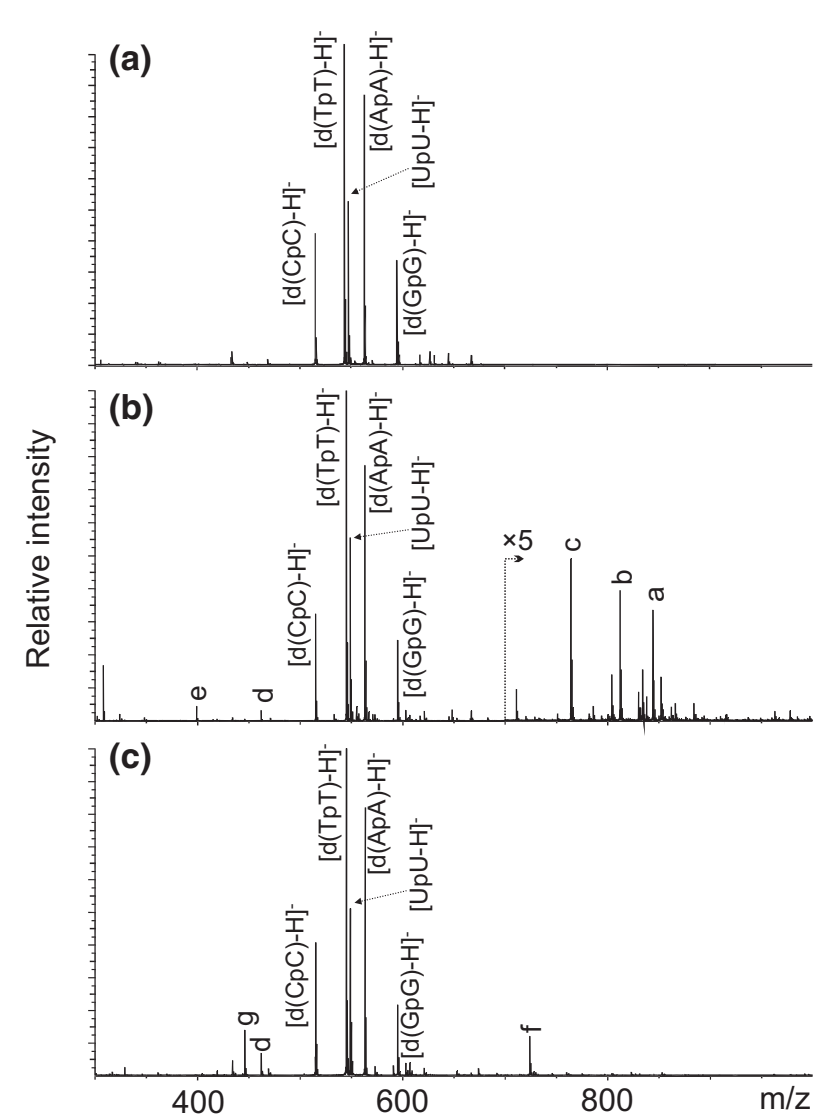

Figure 1. (a) Standard dinucleotide mixture in $10 \mathrm{mM}$ ammonium acetate ( $\mathrm{pH} 8.0$ ); (b) CHB-dinucleotide reaction mixture after $24 \mathrm{~h}$ at $37^{\circ} \mathrm{C}$; (c) sTT-dinucleotide reaction mixture after $6 \mathrm{~h}$ at $37^{\circ} \mathrm{C}$. All spectra were obtained by nanospray-FTMS in negative ion mode (see the Experimental section). In panel (b), Species a corresponds to the $\mathbf{C H B}$ monofunctional adduct of $\mathrm{d}(\mathrm{GpG})$ with hydrolyzed 2-chloroethyl function; $\mathbf{b}$ and $\mathbf{c}$ are analogous adducts of $\mathrm{d}(\mathrm{ApA})$ and $\mathrm{d}(\mathrm{CpC}) ; \mathbf{d}$ is the species formed by hydrolytic depurination of $d(G p G)$; $e$ is the depurination product $\mathrm{N}-(7-$ guaninylethyl)-N-hydroxyethyl-p-acetaminophenylbutyric acid. In panel (c), Species $\mathbf{f}$ is the sTT monofunctional adduct of $d(G p G)$ with hydrolyzed $\mathrm{Cl} ; \mathbf{d}$ the species formed by hydrolytic depurination of $\mathrm{d}(\mathrm{GpG})$ [observed also in panel (b)]; $\mathbf{g}$ is the analogous depurinated $\mathrm{d}(\mathrm{ApA})$.

internal standards [in this case $\mathrm{d}(\mathrm{TpT})$ and UpU], we found that a $48 \% \pm 4 \%$ of initial $\mathrm{d}(\mathrm{GpG})$ had reacted with $\mathrm{CHB}$ after $24 \mathrm{~h}$ at $37^{\circ} \mathrm{C}$, while $38 \% \pm 6 \%$ of initial $\mathrm{d}(\mathrm{ApA})$ and $23 \% \pm 5 \% \mathrm{~d}(\mathrm{CpC})$ were transformed under the selected experimental conditions (based on five repeated reactions).

The possibility that each substrate may undergo transformation according to different reaction pathways (e.g., formation of multiple products and possible degradation, vide infra) is clearly not accounted for by this semiquantitative approach, which nevertheless proved to be very valuable in guiding the optimization of critical parameters for actual probing applications. The percentage of substrate transformation was employed to evaluate the effects of buffer solution, probe to substrate ratio, and incubation time on the crosslinking of di- and trinucleotide standards. Considering the 
Table 1. Characteristics of candidate bifunctional probes

\begin{tabular}{|c|c|c|c|c|c|c|c|}
\hline Name & $\begin{array}{c}\text { Base } \\
\text { specificity }\end{array}$ & $\begin{array}{l}\Delta \mathrm{m} \\
(\mathrm{Da})^{\mathrm{b}}\end{array}$ & $\begin{array}{l}\text { Adduct } \\
\text { stability }^{\mathrm{c}}\end{array}$ & $\begin{array}{l}\text { Other } \\
\text { products }^{\text {d }}\end{array}$ & $\begin{array}{l}\text { Crosslinking } \\
\text { distance } \\
(\AA)^{\mathrm{e}}\end{array}$ & $\begin{array}{l}\text { Probe/ } \\
\text { substrate }^{f}\end{array}$ & Other reaction conditions \\
\hline BKT & G & 230.079 & + & L & $4.4-6.4$ & $5: 1$ & $\begin{array}{l}20 \mathrm{mM} \text { ammonium borate ( } \mathrm{pH} 8.0), \\
37^{\circ} \mathrm{C}, 2 \mathrm{~h}\end{array}$ \\
\hline PDG & G & 190.027 & + & L & $7.0-8.0$ & $5: 1$ & $\begin{array}{l}20 \mathrm{mM} \text { ammonium borate }(\mathrm{pH} 8.0), \\
37^{\circ} \mathrm{C}, 2 \mathrm{~h}\end{array}$ \\
\hline СРТ & $\mathrm{G}>\mathrm{A}>\mathrm{C}$ & 225.995 & +++ & B & 3.2 & $2: 1$ & $\begin{array}{l}10 \mathrm{mM} \text { ammonium cacodylate } \\
\mathrm{pH} 6,37^{\circ} \mathrm{C}, 24 \mathrm{~h}\end{array}$ \\
\hline NM & $\mathrm{G}>\mathrm{A}>\mathrm{C}$ & 83.073 & ++ & $H, D$ & $8.0-11.0$ & $9: 1$ & $\begin{array}{l}10 \mathrm{mM} \text { ammonium } \\
\text { acetate/cacodylate }(\mathrm{pH} 8.0) \\
37^{\circ} \mathrm{C}, 2 \mathrm{~h}\end{array}$ \\
\hline $\mathrm{CHB}$ & $\mathrm{G}>\mathrm{A}>\mathrm{C}$ & 231.126 & ++ & $H, D$ & $8.0-11.0$ & $9: 1$ & $\begin{array}{l}10 \mathrm{mM} \text { ammonium } \\
\text { acetate/cacodylate }(\mathrm{pH} 8.0) \\
37^{\circ} \mathrm{C}, 8 \mathrm{~h}\end{array}$ \\
\hline sTT & G & 110.962 & ++ & $H, D$ & 2.4 & $60: 1$ & $\begin{array}{l}50 \mathrm{mM} \text { ammonium borate, }(\mathrm{pH} \\
6.0), 37^{\circ} \mathrm{C}, 2 \mathrm{~h}\end{array}$ \\
\hline
\end{tabular}

apreferential targets determined under the described conditions (see text for the position of alkylation).

bIncremental mass of bifunctional adduct.

'Stability estimated at the conditions provided: +++ very stable; ++ stable; + somewhat reversible.

${ }^{\mathrm{d} A d d i t i o n a l}$ products: B buffer interaction (e.g., acetate coordination); D depurination; $\mathrm{H}$ hydrolysis of reagent or mono-functional adduct; $\mathrm{L}$ water loss from adduct.

eDistance between crosslinked functions in final adduct. A range is provided for the more flexible reagents.

fThe probe/substrate ratio was calculated in moles of reactive functions in the probe per moles of susceptible sites in the substrate.

complex effects of ionic strength and temperature on the stability of nucleic acid structures, these reaction parameters were more effectively adjusted using structured substrates, on a case by case basis.

Probing reactions were performed using different volatile buffers to test whether analyte solutions could be obtained that would be directly amenable to nanospray-FTMS with no prior desalting or cleanup procedures. Considering the ability manifested by known alkylating agents to react with functional groups present in common biological buffers [49, 50], we looked for the possible formation of adducts between candidate reagents and the MS-friendly buffers included in the study (i.e., ammonium acetate, bicarbonate, borate, and cacodylate, see the Experimental section). No significant signs of direct reactivity were observed under the explored conditions except for CPT, which produced weak coordination complexes with acetate. For this reason, ammonium cacodylate was generally preferred for this reagent to avoid the risk of interfering with its crosslinking activity.

In subsequent experiments, the probe/substrate ratio was adjusted to avoid the saturation of all susceptible sites in the substrate. As discussed later for structured samples, over-probing is prone to structural distortion and may lead to unwanted alkylation of transient conformations that do not provide a fair representation of the structure under investigation. The smaller unstructured standards employed here enabled us to identify probing conditions that would result in only incomplete derivatization of fully exposed bases and, thus, would be less likely to produce artifacts. Once an appropriate probe/substrate ratio was identified, the reaction course was followed as a function of time by analyzing aliquots taken at predetermined intervals. This process allowed for the selection of the best possible incubation time to maximize the formation of products and minimize any possible degradation. In this way, baseline reaction parameters were obtained to allow for direct comparisons between candidate probes and to provide convenient starting conditions for their actual application in structural studies (Table 1).

\section{Target Specificity of Probe Candidates}

The results offered by standard di- and trinucleotide mixtures were also employed to classify the different reagents as base-specific or broad-range, according to their ability to modify individual or multiple bases (Table 1). While nitrogen mustards and CPT were confirmed to target a range of nucleotides, the bis-(1,2dicarbonyls) BKT/PDG and the triazine sTT provided excellent examples of base-specific agents. The latter, in particular was found capable of inducing the exclusive alkylation of $\mathrm{d}(\mathrm{GpG}$ ) (Figure 1c, Species f) through a reaction believed to involve the nucleophilic substitution of N7 to one of the chlorines present in the symmetric molecule (Scheme 1) [47]. The fact that no products were detected for the remaining dinucleotides even after $24 \mathrm{~h}$ incubation at $37^{\circ} \mathrm{C}$ could be explained by the less pronounced nucleophilic character of the functional groups of such bases. On the contrary, the observed specificity of BKT and PDG toward $\mathrm{d}(\mathrm{GpG})$ is consistent with the known reactivity of 1,2-dicarbonyl probes toward guanidino functional groups [51, 52], which effectively excludes the other nucleobases as viable targets. Considering their ability to attack the Watson-Crick interface of guanine (N1 and exocyclic amine), rather than its five-membered ring (Scheme 1), these crosslinkers are expected to provide valuable 
(a)
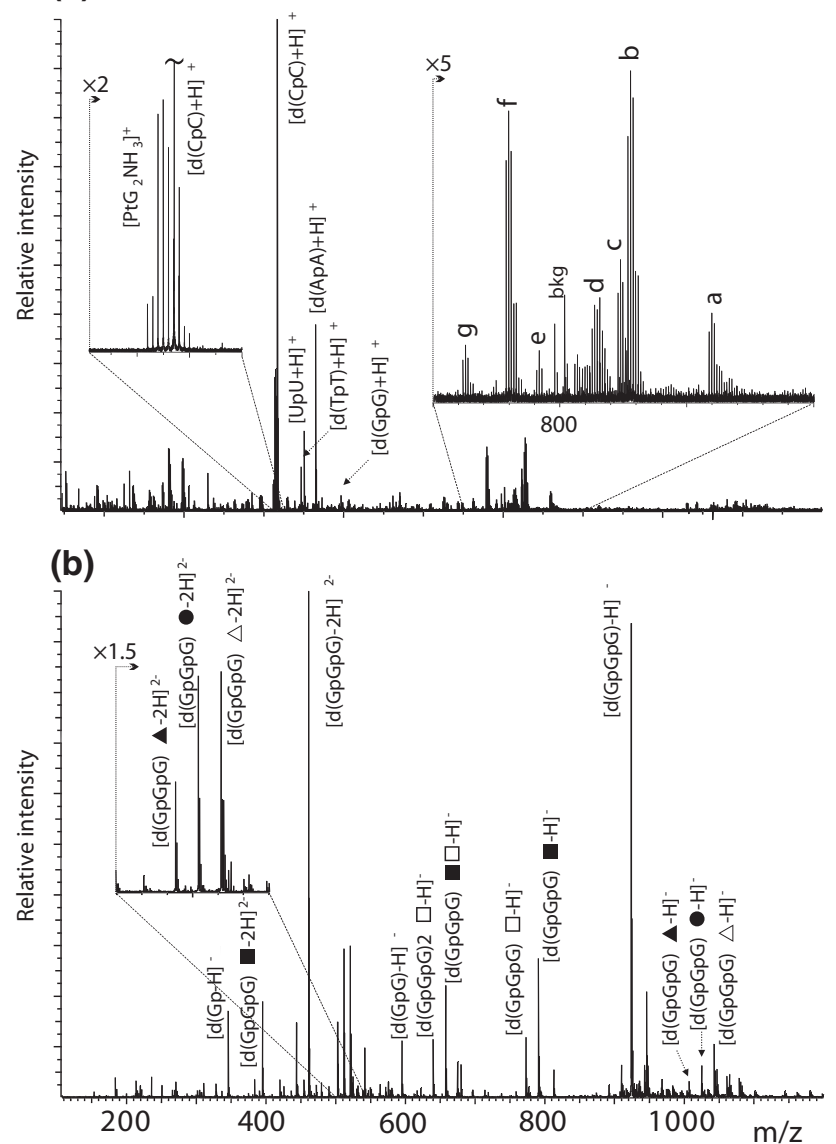

Figure 2. (a) CPT-dinucleotide reaction mixture in $10 \mathrm{mM}$ ammonium cacodylate ( $\mathrm{pH}$ 6.0) after $24 \mathrm{~h}$ at $37^{\circ} \mathrm{C}$; (b) NM$\mathrm{d}(\mathrm{GpGpG})$ reaction mixture in $10 \mathrm{mM}$ ammonium acetate $(\mathrm{pH} 8.0)$ after $2 \mathrm{~h}$ at $37^{\circ} \mathrm{C}$. These spectra were obtained by nanosprayFTMS in positive (a) and negative (b) ion mode (see the Experimental section). In panel (a): Species a corresponds to CPT monofunctional adduct of $\mathrm{d}(\mathrm{GpG}) ; \mathbf{b}$ and $\mathbf{f}$ are the analogous monofunctional adducts of $\mathrm{d}(\mathrm{ApA})$ and $\mathrm{d}(\mathrm{CpC})$; $\mathbf{c}$ and $\mathbf{e}$ are the bifunctional products of $\mathrm{d}(\mathrm{GpG})$ and $\mathrm{d}(\mathrm{ApA})$; $\mathbf{d}$ and $\mathbf{g}$ are hydrolyzed monofunctional products of $\mathrm{d}(\mathrm{ApA})$ and $\mathrm{d}(\mathrm{CpC})$ in which the remaining $\mathrm{Cl}^{-}$is replaced by $\mathrm{H}_{2} \mathrm{O}$. In panel (b): the $\triangle$ corresponds to NM monofunctional adduct of $\mathrm{d}(\mathrm{GpGpG})$; hydrolyzed monofunctional $\left(\mathrm{Cl}^{-}\right.$replaced by $\left.\mathrm{OH}^{-}\right)$; $\mathbf{\Delta}$ bifunctional crosslink; base hydrolysis ( $G$ or modified $G$ formally replaced by $\mathrm{OH}$ ); $\bigcirc$ depurination (elimination of $\mathrm{G}$ or modified G).

complementary information to those obtained by sTT and other N7-alkylating agents.

It is important to note that while nitrogen mustards and sTT did not provide any significant amounts of bridged adducts with standard dinucleotide substrates, crosslinked products were readily observed when similar mixtures were treated with CPT. As shown by samples analyzed in positive ion mode after $24 \mathrm{~h}$ incubation at $37^{\circ} \mathrm{C}$ (Figure 2a), bifunctional adducts of $\mathrm{d}(\mathrm{GpG})$ and $\mathrm{d}(\mathrm{ApA})$ (Species c and e, respectively) were formed in addition to monofunctional products of $\mathrm{d}(\mathrm{GpG}), \mathrm{d}(\mathrm{ApA})$, and $\mathrm{d}(\mathrm{CpC})$ (labeled $a, b$, and $\mathrm{f}$, respectively). These results were ascribed to the ability of CPT to induce the intrastrand crosslinking of contiguous purines through N7 coordination of $\mathrm{Pt}(\mathrm{II})$, while cytosine adducts require the participation of N3 (Scheme 1). Purine-purine crosslinking has been shown to constitute the majority of the observed products in CPT-treated salmon sperm DNA, with guanineguanine alone accounting for up to $47-50 \%$ of the total adducts [53]. These favorable results have been explained by the ability of contiguous purines to assume a "head-to-head" conformation that places the respective N7 positions in the correct situation for effective $\mathrm{Pt}$ (II) bridging [43].

A possible mismatch between the spacing/orientation of the crosslinker's functional groups and the steric situation of the susceptible sites could provide an explanation for the apparent lack of bifunctional products manifested by other probes with dinucleotide substrates. This hypothesis is supported by the observation that analogous mixtures of homo-trinucleotides enabled the prompt formation of bridged products with nitrogen mustards and sTT under otherwise identical experimental conditions. In fact, bifunctional crosslinks of $\mathbf{N M}$ with $\mathrm{d}(\mathrm{pGpGpG)}$ ) were readily detected by nanospray-FTMS in combination with the monofunctional adducts that are typical of nitrogen mustard agents (Figure 2b). Relatively unstructured substrates of larger size present a higher degree of conformational freedom, which is expected to offer increased opportunities for efficient bridging derivatization.

\section{Solution Stability of Alkylated Products}

The direct examination of product solutions with no intervening procedures can provide an excellent assessment of adduct stability in the selected reaction environment, which should minimize unwanted secondary effects and degradation. For example, the direct infusion analysis of samples treated with BKT and PDG suggested the possibility that initial adducts may be rapidly hydrolyzed in the aqueous reaction environment to revert to the initial unmodified structures. The stability of these adducts was greatly increased in borate buffer, as previously observed for products formed by their monofunctional precursor kethoxal [52]. In contrast, adducts provided by CPT, nitrogen mustards, and sTT formed permanent modifications that were relatively stable in the MS-friendly buffers employed in the study. In excellent agreement with the reactivity of these chemicals toward nucleophilic agents, the unreacted crosslinking group present in their monofunctional adducts proved to be susceptible over time to partial hydrolysis. In the case of nitrogen mustards, species containing intact 2-chloroethyl functions were only detected at relatively short reaction times, while longer permanence in solution resulted in their nearly complete transformation into unreactive hydroxyl counterparts (compare for example the CHB reaction after $24 \mathrm{~h}$ with the NM products after $2 \mathrm{~h}$ in Figure $1 b$ and Figure $2 b$, respectively). While hydrolysis 
of the probe itself may compete with the initial alkylation step, deactivation of the remaining 2-chloroethyl group of a monofunctional adduct will certainly preclude the completion of the bridging reaction. Analogous hydrolysis of CPT with formation of aquacomplexes of $\mathrm{Pt}(\mathrm{II})$ (Figure 2a) is not considered as a possible problem, but rather as a necessary condition for the activity of this reagent [43].

An additional effect of the aqueous environment on adduct stability was the possible depurination of modified nucleobases. Species containing abasic lesions produced by hydrolytic attack or base elimination were observed in the reaction mixtures of CPT, nitrogen mustards, and sTT, which share the ability to alkylate the N7-guanine (Scheme 1). These results were consistent with the fact that N7-modification can greatly facilitate the base loss by weakening the $\mathrm{N}$-glycosidic bond and forming an excellent leaving group [54]. Conversely, this process was not observed for adducts of adenine and cytosine with nitrogen mustards, which are known to preferentially modify the N3 positions of these nucleotides (Scheme 1) with no adverse consequences on the N-glycosidic bond. Depending on charging, the complementary species corresponding to the modified nucleobase were also detected in the reaction mixtures, as exemplified by the $\mathrm{N}-(7-$ guaninylethyl)-N-hydroxyethyl-p-acetaminophenylbutyric acid observed in Figure $1 b$ (Species e).

In addition to base losses that are clearly attributed to chemical modification, indirect effects connected to the presence of crosslinker in solution were noted in the sTT reaction. In fact, abasic $\mathrm{d}(\mathrm{ApA})$ was detected in dinucleotide mixtures treated with this reagent in 10 $\mathrm{mM}$ ammonium borate at $37^{\circ} \mathrm{C}$ for $6 \mathrm{~h}$, despite the fact that this substrate is not susceptible to sTT attack (Figure 1c, Species g). A proposed explanation involved the possibility that the hydrolysis of this molecule with a high content of replaceable chlorine (Scheme 1) could lead to the formal release of enough hydrochloric acid to overwhelm the buffer capacity under the initial test conditions. A lower $\mathrm{pH}$ could then induce the N7protonation of unmodified adenine and guanine nucleotides, which favors purine hydrolysis. This hypothesis received further support by the observation that increasing the concentration of ammonium borate to above $50 \mathrm{mM}$ proved sufficient to alleviate the problem. It is important to note that buffer concentration (in addition to $\mathrm{pH}$ ) should be carefully adjusted according to the amount of hydrolyzable crosslinker employed in the actual probing reaction.

\section{Tandem Mass Spectrometry of Probe Adducts}

As part of bottom-up strategies involving nuclease digestion to locate probed nucleotides in the context of large constructs, gas-phase sequencing is often necessary to resolve possible ambiguities arising from the presence of multiple susceptible bases in the same
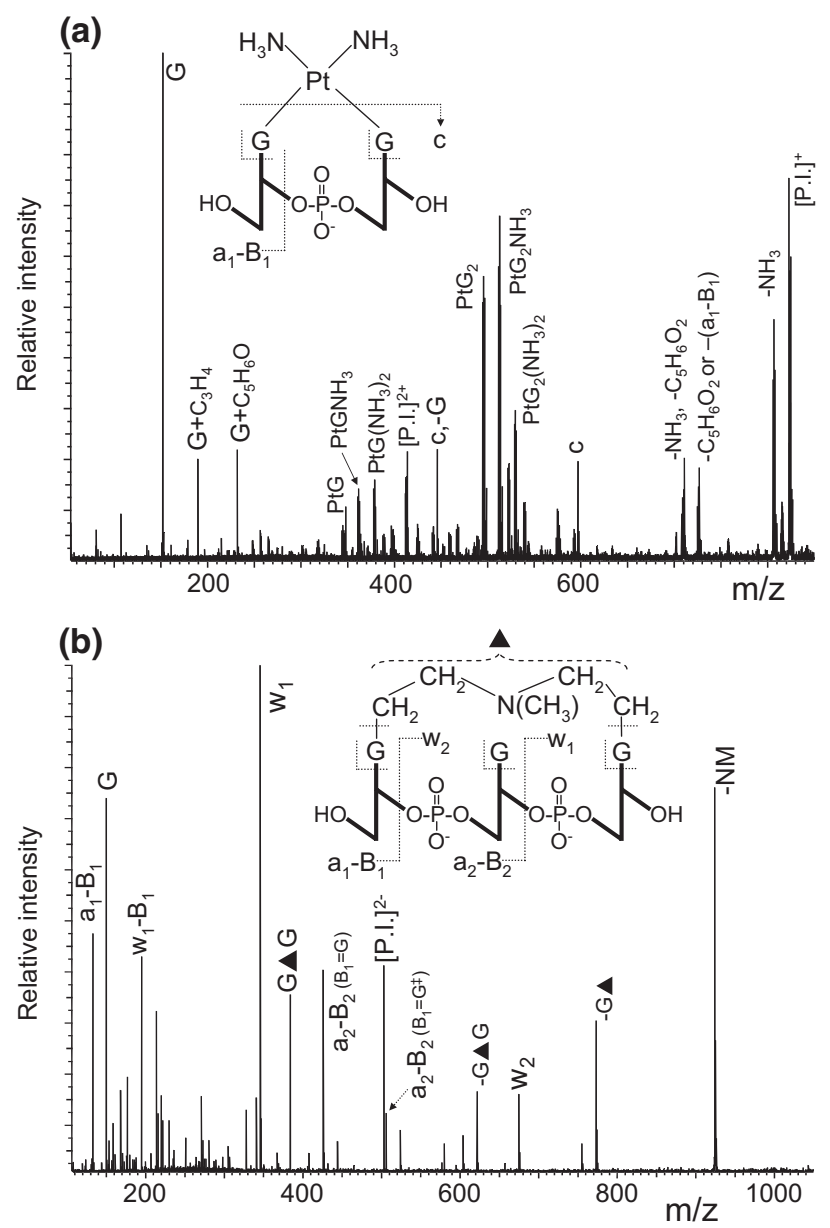

Figure 3. (a) SORI-CID of singly-charged CPT-d(GpG) bifunctional adduct in positive ion mode; (b) SORI-CID of doublycharged NM-d(GpGpG) bifunctional adduct in negative ion mode (see the Experimental Section). Unless specified in the figure, all ions were singly-charged. Ion series were assigned according to standard nucleic acid nomenclature [57]. Labels preceded by dash indicate loss from the precursor ion (P.I.) or preceding species; labels separated by a comma indicate multiple cleavages or losses.

hydrolytic product. Alternatively, tandem mass spectrometry can be applied directly to probed substrates according to top-down approaches that dispense with the hydrolysis step. For this reason, precious information about adduct stability in the gas phase is necessary to guide the experimental design and aid the data interpretation. Mono- and bifunctional adducts of small nucleic acids constitute very convenient models to investigate fragmentation patterns and enable direct comparisons between reagents.

Subjected to SORI-CID [38] (see the Experimental section), the bifunctional CPT-adduct of $d(G p G)$ provided products consistent with those expected from unmodified dinucleotides $[55,56]$ and oligonucleotides [57], as well as others that were a clear reflection of the adduct structure (Figure 3a). In fact, fragments corresponding to adduct ejection (Species $\mathrm{c}$ ) with subsequent base loss (Species $\mathbf{c},-\mathbf{G}$ ) and products including gua- 

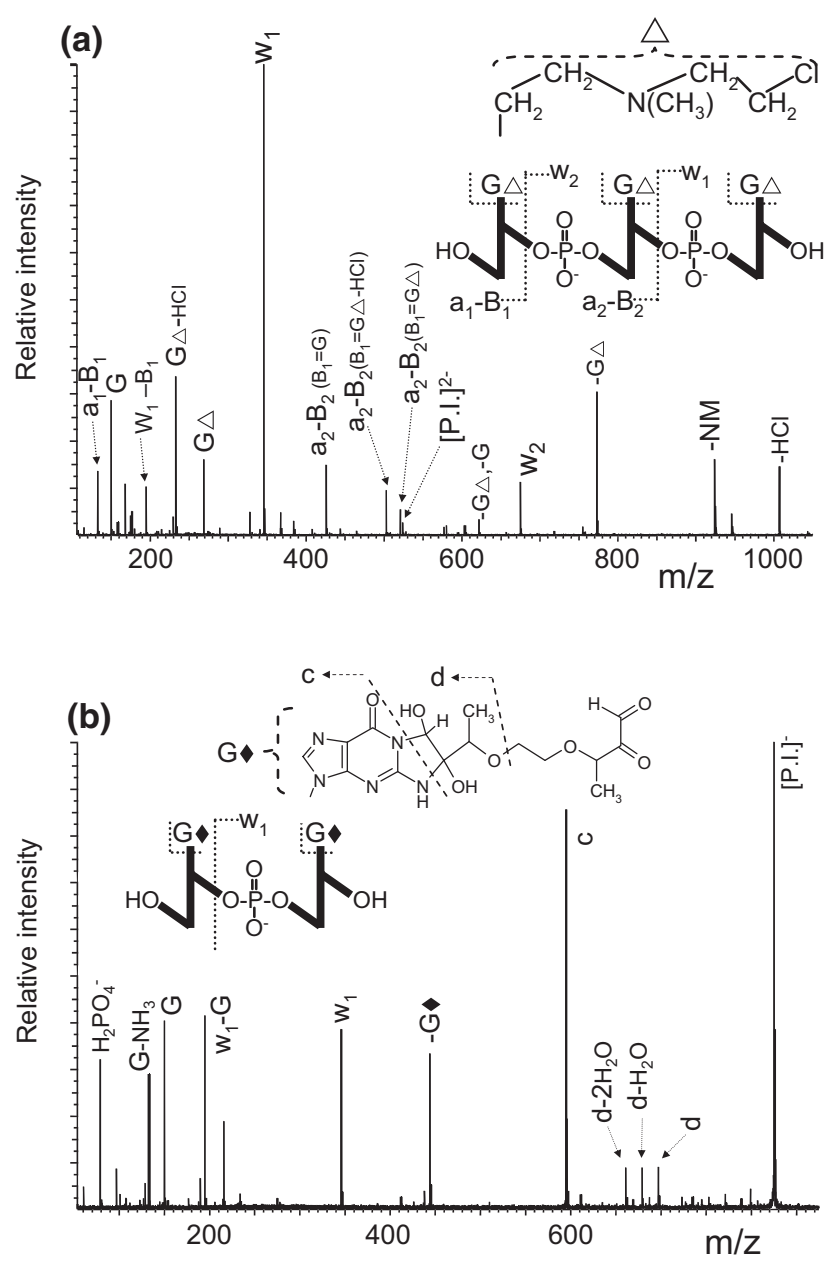

Figure 4. (a) SORI-CID of doubly-charged NM-d(GpGpG) monofunctional adduct; (b) SORI-CID of singly-charged BKT$\mathrm{d}(\mathrm{GpG})$ monofunctional adduct (see the Experimental section). Unless specified in the figure, all ions were singly-charged anions. Ion series were assigned according to standard nucleic acid nomenclature [57]. Labels preceded by dash indicate loss from the precursor ion (P.I.) or preceding species; labels separated by a comma indicate multiple cleavages or losses.

nine and parts of the deoxyribose moiety $\left(\mathrm{G}+\mathrm{C}_{5} \mathrm{H}_{6} \mathrm{O}\right.$, $\mathrm{G}+\mathrm{C}_{3} \mathrm{H}_{4}$ and $\mathrm{G}$ ) could be directly correlated to the initial nucleic acid structure. Species containing Pt(II) and different combinations of guanine and ammonia ligands were a clear result of metal coordination, which can weaken the N-glycosidic bond and facilitate its gas-phase cleavage [58-60]. In addition, products corresponding to the loss of furanyl fragments $\left[-\mathrm{C}_{5} \mathrm{H}_{6} \mathrm{O}_{2}\right.$, or $\left.-\left(\mathbf{a}_{1}-\mathbf{B}_{1}\right)\right]$ were explained by combining the effects of typical backbone cleavages with the ability of $\mathrm{Pt}(\mathrm{II})$ to bridge between the resulting moieties.

Characteristic fragments comprising crosslinked structures were also obtained by gas-phase activation of bifunctional adducts of other candidate reagents. A species corresponding to two bridged guanine bases $(\mathrm{G} \Delta \mathrm{G})$ and its complementary product with two abasic lesions $(-G \mathbf{\Delta} G)$ were readily detected in the SORI-CID spectrum of NM-crosslinked d(GpGpG) (Figure 3b). The nucleic acid backbone of this product offered sequence ions [57] that located the alkylated sites in position 1 and 3 of the trinucleotide. In contrast, no bridged products were obtained from the corresponding monofunctional adduct (Figure 4a), which instead produced characteristic fragments linked to the presence of an unreacted 2-chloroethyl function (e.g., elimination of $\mathrm{HCl}$ ). The observed sequence ions were in this case consistent with the possible alkylation of any of the trinucleotide positions, as expected from the absence of significant steric considerations that may favor one site over the others.

The results provided by bis-(1,2-dicarbonyl)-adducts were largely affected by the fragile nature of the bonding between base and modifier already noticed in solution. The normal cleavages of the nucleic acid backbone were immediately recognizable in the SORICID spectrum of BKT-d(GpG) (Figure $4 b$ ), but the facile dissociation of the alkylating group with restoration of the initial dinucleotide structure was the most prominent process even under gentle activation conditions (Species c). Despite the fact that BKT alkylation does not involve the five-membered ring and should not directly affect the $\mathrm{N}$-glycosidic bond, the modified base was readily lost and the complementary product with one abasic lesion was detected only with retention of an unmodified nucleobase (Species $-\mathrm{G} \downarrow$ ). Weaker signals produced by the loss of 2-keto-3-ethoxybutyraldehyde (Species d) with subsequent dehydration were clearly attributed to a partially cleaved bridging structure, thus indicating that the outright dissociation of the modifying group was not quantitative. This information and the absence of bridged fragments cast doubt on the actual bifunctional nature of the product investigated in this experiment, which was compounded by the fact that the completion of the bridging reaction would not involve a net mass change over the monofunctional product. For these reasons, a putative monofunctional structure was assigned to this species, with the BKT modifier possibly located on either nucleotide. In actual structural applications, this type of ambiguity would be fortunately limited to BKT/PDG crosslinks involving contiguous guanines that could not be adequately segregated on different hydrolytic products by nuclease digestion. In general, the crosslinking of contiguous positions of the target sequence is not as informative as that of distal bases, which is a direct reflection of the 3D structure of the construct of interest.

In addition to providing helpful indications for recognizing the bifunctional nature of probed species (e.g., detection of bridged bases and related fragments), these observations clearly point toward their gas-phase stability as the single most important factor for predicting the ability to identify their position by tandem mass spectrometry. Conjugated products obtained from probes that induce facile base loss are more likely to produce spectra dominated by depurinated fragments 
with abasic lesions corresponding to the crosslinked positions. Subsequent isolation and activation of such fragments in $\mathrm{MS}^{\mathrm{n}}$ experiments are expected to yield sufficient information to locate the abasic sites on the substrate sequence [7]. Unfortunately, this strategy is expected to fail when crosslinking is produced by reagents that are prone to complete adduct dissociation. In this case, the first generation fragment would correspond to an unmodified oligonucleotide that bears no "memory" of the actual position of the bridged bases. For this type of reagent, the only viable solution would involve the classic bottom-up strategy in which the crosslinked substrate is subjected to strand hydrolysis by complementary nucleases.

\section{Crosslinking of Structured RNA}

Candidate bifunctional probes were also tested for their ability to provide meaningful spatial constraints by using RNA substrates of well characterized structure. For efficient bridging derivatization to occur, suitable constructs must include functional groups that are accessible to the probe and are placed within proper distance and orientation. Due to the presence of exposed single-stranded loops and more protected double-stranded stems, stemloop motifs present an ideal combination of steric situations for bifunctional crosslinking. For example, an excellent test substrate was provided by the 37-mer mutant of HIV-1 stemloop 1 (SL1A, see the Experimental section), which is responsible for the dimerization of genomic RNA during the replication of the AIDS virus [61,62]. This construct includes a relatively large and flexible loop, as well as a smaller and more constrained internal bulge, which were found to be readily crosslinkable in vitro under experimental conditions identified using the unstructured standards. In this case, however, the position of alkylated nucleotides was identified according to a bottom-up strategy based on specific nuclease digestion.

The results obtained by treating SL1A with CPT and NM are compared in Figure 5 (see the Experimental section). The bifunctional products identified by RNase A mapping and tandem sequencing are summarized in Tables 2 and 3 and visualized on the secondary struc-

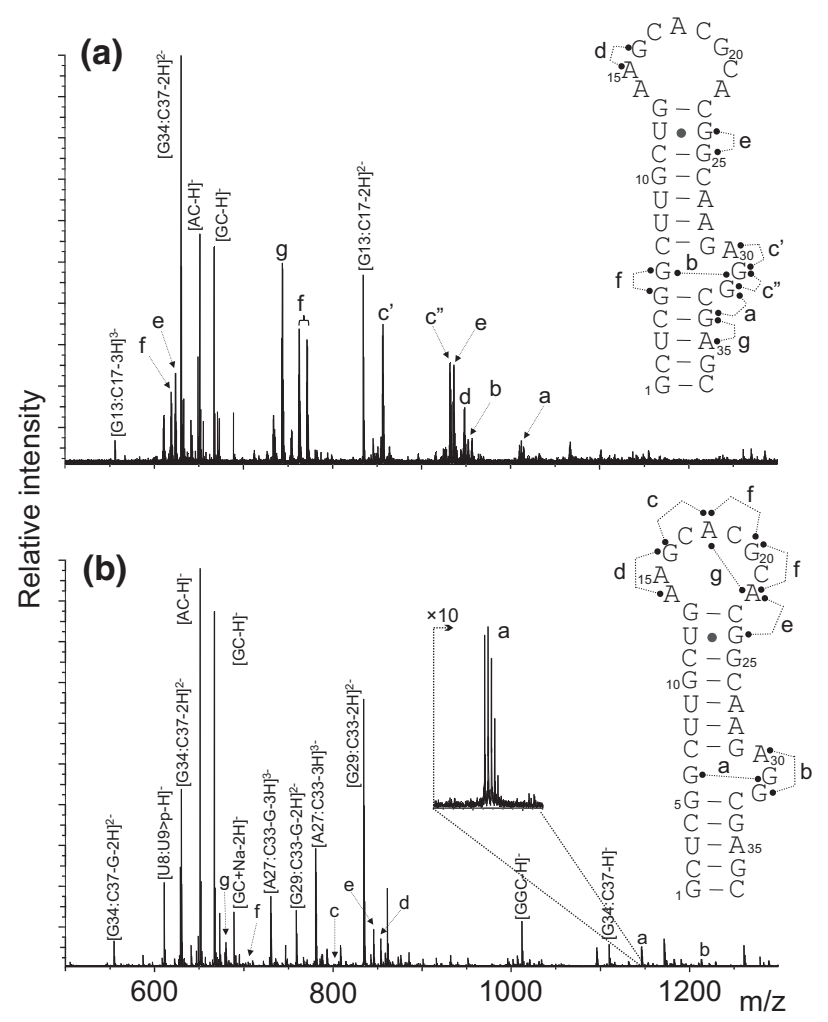

Figure 5. RNase A digestion map of HIV-1 SL1A after probing with CPT (a) and NM (b) (see the Experimental section for the reaction conditions). Oligonucleotide products are indicated by the first and last base separated by a colon. Cyclic phosphate is indicated by the "greater than" $(>)$ symbol. Letters identify major crosslinked products, which are reported on the hairpin secondary structures and cross-referenced in Tables 2 and 3. Species $\mathbf{c}^{\prime}$ and $\mathbf{c}^{\prime \prime}$ include one and two CPT adducts within the same digestion product.

ture of the bulged hairpin. These data clearly reflect the fact that while these reagents share similar basespecificity profiles, the nature of their respective products is very different. Consistent with the relatively short spacing necessary for $\mathrm{Pt}(\mathrm{II})$ coordination, the majority of CPT adducts involved purines present in immediately contiguous positions of single- as well as double-stranded stretches. The latter appeared to concentrate in regions where the regular base stacking was disrupted either by noncanonic base pairing, or by

Table 2. Summary of crosslinked products obtained by treating HIV-1 SL1 with CPT, followed RNase A mapping. Bridged nucleotides are in bold. Product labels refer to Figure 5a

\begin{tabular}{llrr}
\hline Crosslinked products & \multicolumn{1}{c}{ Sequence } & Expected mass & Observed mass \\
\hline \hline A27:C33-G34:C37 & AAGAGGCp-GAGC + CPT & 4059.58 & 4060.79 \\
G5:C7-A27:C33 & GGCp-AAGAGGCp + CPT & 3810.49 & 3810.68 \\
A27:C33 (internal) & AAGAGGCp + CPT & 2571.35 & 2571.47 \\
& AAGAGGCp + 2CPT & 2797.35 & 2797.49 \\
G13:C17 (internal) & GAAGCp + CPT & 1897.25 & 1897.35 \\
A22:C26 (internal) & ACGGCp + CPT & 1873.24 & 1873.33 \\
C4:C7 (internal) & CGGCp + CPT & 1544.26 & 1544.19 \\
G34:C37 (internal) & GAGC + CPT & 1488.23 & 1488.30 \\
G5:C7 (internal) & GGCp + CPT & 1239.14 & 1239.19 \\
\hline
\end{tabular}


Table 3. Summary of crosslinked products obtained by treating HIV-1 SL1 with NM, followed RNase A mapping (see Experimental Section). Bridged nucleotides are in bold. Product labels refer to Figure $5 \mathrm{~b}$

\begin{tabular}{llcr}
\hline \multicolumn{1}{c}{ Crosslinked products } & \multicolumn{1}{c}{ Sequence } & Expected mass & Observed mass \\
\hline \hline A27:C33-G5:C7 & AAGAGGCp-GGCp + NM & 3441.57 & 3441.56 \\
A27:C33 (internal) & AAGAGGCp + NM & 2428.42 & 2428.43 \\
G13:C19 (internal) & GAAGCACp + NM & 2406.43 & 2406.43 \\
G13:C17 (internal) & GAAGCp + NM & 1754.32 & 1754.33 \\
A22:C23-G24:C26 & ACp-GGCp + NM & 1730.31 & 1730.32 \\
G20:C21-A18:C19/A22:C23 & GCp-ACp + NM & 1403.27 & 1403.28 \\
A18:C19-A22:C23 & ACp-ACp + NM & 1369.27 & b \\
\hline
\end{tabular}

possible strand "breathing" near the loop and bulge. In contrast, NM was found to bridge mostly singlestranded purines located across loops or in RNR positions (where $\mathrm{R}=$ purine and $N=$ any nucleotide), which is consistent with the longer spacing between reactive groups typical of nitrogen mustard agents.

The majority of the observed crosslinks were found to satisfy the actual distance and spatial orientation presented by the bridged nucleotides in the high-resolution structures available for this hairpin in the Protein Data Bank $[63,64]$. In a few cases, however, the ability to undergo crosslinking could be explained by invoking the slight rotation of a nucleobase about its N-glycosidic bond, or the possible backbone flexibility in a single-stranded region. As alluded to earlier, the possibility that this approach may be sensitive to transient conformations and kinetic traps should always be contemplated when translating crosslinking information into actual spatial constraints. Although an exhaustive discussion of this topic is beyond the scope of this report, there are a few precautions that should be taken at the experimental level to minimize this risk, or at least recognize the formation of artifacts. In general, over-probing should be avoided by keeping the probe/substrate ratio as low as possible and by striving to achieve ideal "single-hit" conditions (each substrate molecule should be modified only once, but different positions may be hit on different molecules). This requirement is the reason for the usually low abundances manifested by crosslinked products, which place greater demands on the sensitivity and dynamic range of the analytical platform of choice. The baseline conditions identified using standard substrates constitute a very helpful starting point for the optimization of probe/ substrate ratio and other parameters in the context of the actual construct under investigation. In our hands, the ratios that were eventually applied to structured substrates were generally lower than those originally identified using fully exposed unstructured standards. Depending on the specific structural characteristics of the construct of interest, probing should be repeated after introducing small variations of ionic strength, $\mathrm{pH}$, and temperature, which are expected to have more dramatic effects on relatively unstable transient conformations than on stable folded structures [4, 7]. A higher confidence level should be placed on reproducible and moderately abundant crosslinks, which would reflect unchanged accessi- bility and favorable target orientation despite the changing environmental conditions.

\section{Conclusions}

Important aspects of crosslinking activity should be carefully examined before prospective bifunctional probes can be effectively employed in actual structural investigations. Many of these aspects can be conveniently evaluated using simple mixtures comprising dinucleotides and trinucleotides of known composition, which enabled us to dispense with sample cleanups or separation procedures before direct analysis by nanospray-FTMS. Following this approach, adducts formed by mono- and bifunctional alkylation, as well as characteristic degradation products, were immediately identified and characterized by taking advantage of the reduced complexity presented by these reaction mixtures. The preferential activity of the investigated crosslinkers toward selected targets was immediately revealed by the competition between different substrates of homogenous base composition with no need for nuclease digestion or sequencing methods.

Among the candidate probes, the bis-(1,2-dicarbonyl) and triazine derivatives were proven to be exclusively guanine-specific under the selected conditions. The different position of their respective attack is expected to provide valuable complementary information about the structural situation of the probed bases. In contrast, the nitrogen mustards and coordinative agents have shown to possess a broader and nearly overlapping range of targets, but the actual nature of the bridged products was found to be narrowly defined by the structural context of the susceptible bases.

In future work, the described methods will be employed to test additional bifunctional agents with different reactivity profiles. While a few crosslinkers are available for purines and cytosine, there are no convenient crosslinkers for uridine and thymine. The possibility of obtaining bridging modifications between functional groups on the phospho-pentose backbone should also be investigated to increase the number and type of spatial constraints attainable by this approach. As new crosslinkers will be added to the available tool kit, MS3D will become increasingly more appealing for 
the structural elucidation of substrates that are not readily amenable to the traditional techniques employed in structural biology.

\section{Acknowledgments}

This research was funded by the National Institutes of Health (R01-GM643208) and the National Science Foundation (CHE0439067).

\section{References}

1. Young, M. M.; Tang, N.; Hempel, J. C.; Oshiro, C. M.; Taylor, E. W.; Kuntz, I. D.; Gibson, B. W.; Dollinger, G. High throughput protein fold identification by using experimental constraints derived from intramolecular crosslinks and mass spectrometry. Proc. Natl. Acad. Sci. U.S.A. 2000, 97, 5802-5806.

2. Back, J. W.; de Jong, L.; Muijsers, A. O.; de Koster, C. G. Chemical crosslinking and mass spectrometry for protein structural modeling. J. Mol. Biol. 2003, 331, 303-313.

3. Sinz, A. Chemical cross-linking and mass spectrometry for mapping three-dimensional structures of proteins and protein complexes. J. Mass Spectrom. 2003, 38, 1225-1237.

4. Yu, E.; Fabris, D. Direct probing of RNA structures and RNA-protein interactions in the HIV-1 packaging signal by chemical modification and electrospray ionization Fourier transform mass spectrometry. J. Mol. Biol. 2003, 330, 211-223.

5. Pancerella, C.; Leahy, D.; Yang, C.; Young, M.; Sale, K.; Rahn, L.; Kuntz, I.; Fabris, D. Data portal enabling new protein structure collaboration. Proceedings of the 19th Protein Society Meeting; Boston, MA, August, 2005

6. Yu, E. T.; Fabris, D. Toward multiplexing the application of solvent accessibility probes for the investigation of RNA three-dimensional structures by electrospray ionization-Fourier transform mass spectrometry. Anal. Biochem. 2004, 344, 356-366.

7. Kellersberger, K. A.; Yu, E.; Kruppa, G. H.; Young, M. M.; Fabris, D. Top-down characterization of nucleic acids modified by structural probes using high-resolution tandem mass spectrometry and automated data interpretation. Anal. Chem. 2004, 76, 2438-2445.

8. Yu, E. T.; Zhang, Q.; Fabris, D. Untying the FIV frameshifting pseudoknot structure by MS3D. J. Mol. Biol. 2005, 345, 69-80.

9. Zwieb, C.; Ross, A.; Rinke, J.; Meinke, M.; Brimacombe, R. Evidence for RNA-RNA cross-link formation in Escherichia coli ribosomes. Nucleic Acids Res. 1978, 5, 2705-2720.

10. Mitchell, P.; Osswald, M.; Brimacombe, R. Identification of intermolecular RNA cross-links at the subunit interface of the Escherichia coli ribosome. Biochemistry 1992, 31, 3004-3011.

11. McCloskey, J. A.; Crain, P. F. Progress in mass spectrometry of nucleic acid constituents: Analysis of xenobiotic modifications and measurements at high mass. Int. J. Mass Spectrom. Ion Processes 1992, 118/119, 593-615.

12. Farmer, P. B.; Sweetman, G. M. A. Mass spectrometric detection of carcinogen adducts. J. Mass Spectrom. 1995, 30, 1369-1379.

13. Barry, J. P.; Vouros, P.; Van Schepdael, A.; Law, S.-J. Mass and sequence verification of modified oligonucleotides using electrospray tandem mass spectrometry. J. Mass Spectrom. 1995, 30, 993-1006.

14. Nordhoff, E.; Kirpekar, F.; Roepstorff, P. Mass spectrometry of nucleic acids. Mass Spectrom. Rev. 1996, 15, 67-138.

15. Deforce, D. L.; Ryniers, F. P.; van den Eeckhout, E. G.; Lemiere, F.; Esmans, E. L. Analysis of DNA adducts in DNA hydrolysates by capillary zone electrophoresis and capillary zone electrophoresiselectrospray mass spectrometry. Anal. Chem. 1996, 68, 3575-3584.

16. Chaudhary, A. K.; Reddy, G. R.; Blair, I. A.; Marnett, L. J. Characterization of a N6-oxopropenyl-2'-deoxyadenosine adduct in malondialdehyde-modified DNA using liquid chromatography/ electrospray ionization tandem mass spectrometry. Carcinogenesis 1996, $17,1167-1170$

17. Iannitti, P.; Sheil, M. M.; Wickham, G. High sensitivity and fragmentation specificity in the analysis of drug-DNA adducts by electrospray tandem mass spectrometry. J. Am. Chem. Soc. 1997, 119, 1490-1491.

18. Kirpekar, F.; Krogh, T. N. RNA fragmentation studied in a matrixassisted laser desorption/ionization tandem quadrupole/orthogonal time-of-flight mass spectrometer. Rapid Commun. Mass Spectrom. 2001, $15,8-14$.

19. Beck, J. L.; Colgrave, M. L.; Ralph, S. F.; Sheil, M. M. Electrospray ionization mass spectrometry of oligonucleotide complexes with drugs, metals, and proteins. Mass Spectrom. Rev. 2001, 20, 61-87.

20. Fenn, J. B.; Mann, M.; Meng, C. K.; Wong, S. F.; Whitehouse, C. M Electrospray ionization for mass spectrometry of large biomolecules. Science 1989, 246, 64-71.

21. Comisarow, M. B.; Marshall, A. G. Fourier transform ion cyclotron resonance. Chem. Phys. Lett. 1974, 25, 282-283.

22. Hendrickson, C. L.; Emmett, M. R.; Marshall, A. G. Electrospray ionization Fourier transform ion cyclotron resonance mass spectrometry. Annu. Rev. Phys. Chem. 1999, 50, 517-536.
23. Woodin, R. L.; Bomse, D. S.; Beauchamp, J. L., Multiphoton dissociation of molecules with low power continuous wave infrared laser radiation. J. Am. Chem. Soc. 1978, 100, 3248-3250.

24. Little, D. P.; Speir, J. P.; Senko, M. W.; O'Connor, P. B.; McLafferty, F. W. Infrared multiphoton dissociation of large multiply charged ions for biomolecule sequencing. Anal. Chem. 1994, 66, 2809-2815.

25. Zubarev, R. A.; Kelleher, N. L.; McLafferty, F. W. Electron capture dissociation of multiply charged protein cations: A nonergodic process. J. Am. Chem. Soc. 1998, 120, 3265-3266.

26. Zubarev, R. A.; Horn, D. M.; Fridriksson, E. K.; Kelleher, N. L.; Kruger, N. A.; Lewis, M. A.; Carpenter, B. K.; McLafferty, F. W. Electron capture dissociation for structural characterization of multiply charged protein cations. Anal. Chem. 2000, 72, 563-573.

27. Kruppa, G. H.; Schoeniger, J.; Young, M. M. A top down approach to protein structural studies using chemical cross-linking and Fourier transform mass spectrometry. Rapid Commun. Mass Spectrom. 2003, 17, 155-162.

28. Novak, P.; Kruppa, G. H.; Young, M. M.; Schoeniger, J., A top down method for the determination of residue specific solvent accessibility in proteins. J. Mass Spectrom. 2004, 39, 322-328.

29. Wilm, M.; Mann, M. Analytical properties of the nanoelectrospray ion source. Anal. Chem. 1996, 68, 1-8.

30. Ehresmann, C.; Baudin, F.; Mougel, M.; Romby, P.; Ebel, J.-P.; Ehresmann, B. Probing the structure of RNAs in solution. Nucleic Acids Res. 1987, 12, 9109-9128.

31. Expert-Bezançon, A.; Chiaruttini, C. RNA-protein crosslinking. Methods Enzymol. 1988, 164, 310-318.

32. Brimacombe, R.; Stiege, W.; Kyriatsoulis, A.; Maly, P. Intra-RNA and RNA-protein crosslinking techniques in Escherichia coli ribosomes. Methods Enzymol. 1988, 164, 287-309.

33. Rajski, S. R.; Williams, R. M. DNA crosslinking agents as antitumor drugs. Chem. Rev. 1998, 98, 2733-2795.

34. Luce, R. A.; Hopkins, P. B. Chemical crosslinking of drugs to DNA. Methods Enzymol. 2001, 340, 396-421.

35. Brewer, L. A.; Goelz, S.; Noller, H. F. Ribonucleic acid-protein crosslinking within the intact Escherichia coli ribosome, utilizing ethylene glycol bis[3-(2-ketobutyraldehyde)ether], a reversible bifunctional reagent: Synthesis and crosslinking within $30 \mathrm{~S}$ and $50 \mathrm{~S}$ subunits. Biochemistry 1983, 22, 4303-4309.

36. Milligan, J. F.; Groebe, D. R.; Witherell, G. W.; Uhlenbeck, O. C. Oligoribonucleotide synthesis using T7 RNA polymerase and synthetic DNA templates. Nucleic Acids Res. 1987, 15, 8783-8798.

37. de Koning, L. J.; Nibbering, N. M. M.; van Orden, S. L.; Laukien, F. H. Mass selection of ions in a Fourier transform ion cyclotron resonance trap using correlated harmonic excitation fields (CHEF). Int. J. Mass Spectrom. Ion Processes 1997, 165/166, 209-219.

38. Gauthier, J.W.; Trautman, T. R.; Jacobson, D. B. Sustained off-resonance irradiation for collision-activated dissociation involving Fourier transform mass spectrometry. Collision-activated dissociation technique that emulates infrared multiphoton dissociation. Anal. Chim. Acta. 1991, $246,221-225$

39. Senko, M. W.; Speir, J. P.; McLafferty, F. W. Collisional activation of large multiply charged ions using Fourier transform mass spectrometry. Anal. Chem. 1994, 66, 2801-2808.

40. Staehelin, M. Inactivation of virus nucleic acids with glyoxal derivatives. Biochim. Biophys. Acta. 1959, 31, 448-454.

41. Noller, H. F.; Chaires, J. B. Functional modification of $16 \mathrm{~S}$ ribosomal RNA by kethoxal. Proc. Nat. Acad. Sci. U.S.A. 1972, 69, 3115-3118.

42. Lippard, S. J. New chemistry of an old molecule: cis-[Pt(NH3)2Cl2]. Science 1982, 218, 1075-1082.

43. Jamieson, E. R.; Lippard, S. J. Structure, recognition, and processing of cisplatin-DNA adducts. Chem. Rev. 1999, 99, 2467-2498.

44. Brookes, P.; Lawley, P. D. The reaction of mono- and difunctional alkylating agents with nucleic acids. Biochem. J. 1961, 80, 496-503.

45. Lawley, P. D.; Brookes, P. Molecular mechanism of the cytotoxic action of difunctional alkylating agents and of resistance to this action. Nature $1965,206,480-483$

46. Kundu, G. C.; Schullek, J. R.; Wilson, I. B. The alkylating properties of chlorambucil. Pharmacol. Biochem. Behavior 1994, 49, 621-624.

47. Oste, C.; Brimacombe, R. The use of sym-triazine trichloride in RNAprotein cross-linking studies with Escherichia coli ribosomal subunits. Mol. Gen. Genet. 1979, 168, 81-86.

48. Null, A. P.; Nepomuceno, A. I.; Muddiman, D. C. Implications of hydrophobicity and free energy of solvation for characterization of nucleic acids by electrospray ionization mass spectrometry. Anal. Chem. 2003, 75, 1331-1339.

49. Richter, S.; Fabris, D.; Binaschi, M.; Gatto, B.; Capranico, G.; Palumbo, M. Effects of common buffer systems on drug activity: The case of clerocidin. Chem. Res. Toxicol. 2004, 17, 492-501.

50. Richter, S. N.; Fabris, D.; Moro, S.; Palumbo, M. Dissecting reactivity of clerocidin toward common buffer systems by means of selected drug analogs. Chem. Res. Toxicol. 2005, 18, 35-40.

51. Christiansen, J.; Garrett, R. Enzymatic and chemical probing of ribosomal RNA-protein interactions. Methods Enzymol. 1988, 164, 456-468.

52. Akinsiku, O. T.; Yu, E. T.; Fabris, D. Mass spectrometric investigation of protein alkylation by the RNA footprinting probe kethoxal. J. Mass Spectrom. 2005, 40, 1372-1381. 
53. Fichtinger-Schepman, A. M.; van der Veer, J. L.; den Hartog, J. H.; Lohman, P. H.; Reedijk, J. Adducts of the antitumor drug cisdiamminedichloroplatinum(II) with DNA: Formation, identification, and quantitation. Biochemistry 1985, 24, 707-713.

54. Burrows, C. J.; Muller, J. G. Oxidative nucleobase modifications leading to strand scission. Chem. Rev. 1998, 98, 1109-1152.

55. Rodgers, M. T.; Campbell, S.; Marzluff, E. M.; Beauchamp, J. L. Low-energy collision-induced dissociation of deprotonated dinucleotides: Determination of the energetically-favored dissociation pathways and the relative acidities of the nucleic acid bases. Int. J. Mass Spectrom. Ion Processes 1994, 137, 121.

56. Habibi-Goudarzi, S.; McLuckey, S. A. Ion trap collisional activation of the deprotonated deoxymononucleoside and deoxydinucleoside phosphates. J. Am. Soc. Mass Spectrom. 1995, 6, 102-113.

57. McLuckey, S. A.; Habibi-Goudarzi, S. Decompositions of multiply charged oligonucleotide anions. J. Am. Chem. Soc. 1993, 115, 1208512095 .

58. Martin, L. B., III; Schreiner, A. F.; van Breemen, R. B. Characterization of cisplatin adducts of oligonucleotides by fast atom bombardment mass spectrometry. Anal. Biochem. 1991, 193, 6-15.
59. Iannitti-Tito, P.; Weimann, A.; Wickham, G.; Sheil, M. M. Structural analysis of drug-DNA adducts by tandem mass spectrometry. Analyst 2000, 125, 627-633.

60. Hagemeister, T.; Linscheid, M. Mass spectrometry of cis-diamine dichloroplatinum(II) adducts with the dinucleoside monophosphates d(ApG), $\mathrm{d}(\mathrm{GpG})$, and $\mathrm{d}(\mathrm{TpC})$ in an ion trap. J. Mass Spectrom. 2002, 37, 731-747.

61. Darlix, J. L.; Gabus, C.; Nugeyre, M. T.; Clavel, F.; Barre-Sinussi, F. Cis elements and transacting factors involved in the RNA dimerization of the human immunodeficiency virus HIV-1. J. Mol. Biol. 1990, 216, 689-699.

62. Laughrea, M.; Jetté, L. A 19-nucleotide sequence upstream of the 5' major splice donor is part of the dimerization domain of human immunodeficiency virus 1 genomic RNA. Biochemistry 1994, 33, 1346413474.

63. Lawrence, D. C.; Stover, C. C.; Noznitsky, J.; Wu, Z.; Summers, M. F. Structure of the intact stem and bulge of HIV-1 Psi-RNA stem-loop SL1. J. Mol. Biol. 2003, 326, 529-542.

64. Kieken, F.; Arnoult, E.; Barbault, F.; Paquet, F.; Huynh-Dinh, T.; Paoletti, J.; Genest, D.; Lancelot, G.HIV-1(Lai) genomic RNA: Combined use of NMR and molecular dynamics simulation for studying the structure and internal dynamics of a mutated SL1 hairpin. Eur. Biophys. J. 2002, 31, 521-531. 